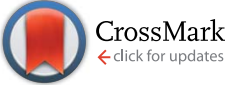

Cite this: RSC Adv., 2017, 7, 12161
Received 18th December 2016 Accepted 15th February 2017

DOI: 10.1039/c6ra28367e

rsc.li/rsc-advances

\title{
Solvent-dependent and highly selective anion sensing and molecular logic application of bisindolylmaleimide derivatives $\uparrow$
}

\author{
Huimei Yao, ${ }^{\mathrm{a}}$ Jingwei Wang, ${ }^{\mathrm{a}}$ Huan Chen, ${ }^{\mathrm{b}}$ Xiaofei Mei, ${ }^{\mathrm{b}}$ Zhu Su, ${ }^{\mathrm{a}}$ Jingnan Wu, ${ }^{\mathrm{a}}$ \\ Zhenghuan Lin*a and Qidan Ling*ab
}

\begin{abstract}
A series of bisindolylmaleimide dyes (IMs) with different $N$-substituents (IM-PFB, IM-TBA and IM-MB) and planarity (IMC-MB and $I M-M B)$ were designed and synthesized to detect anions selectively and sensitively. The anion-sensing properties were investigated systematically by changing the $\mathrm{N}$-substituents of maleimide, solvent type and molecular planarity. Results indicate that the anion recognition is significantly affected by the solvent type rather than the $\mathrm{N}$-substituents. Different anion sensitivity in various solvents makes IMs selectively detect $\mathrm{F}^{-}$in $\mathrm{ACN}, \mathrm{H}_{2} \mathrm{PO}_{4}^{-}$in $\mathrm{DCM}$ and $\mathrm{CN}^{-}$in THF. Due to the fixed location of two NH groups, the dye IMC-MB with planar structure exhibits poor sensing selectivity in various solvents. The titration curves of anions show that the sensing mechanism of IMs in various solvents for anions is different. The further experimental and DFT/TDDFT calculation results demonstrate that the hydrogen bond interaction and deprotonation of one $\mathrm{H}$ atom take place in DCM and THF, respectively, and that the two interactions synchronously exist in ACN. Interestingly, the solvent-dependent anion recognition can make IM-PFB mimic the function of three kinds of decoders (1-to-2, 2-to-3 and 2-to-4), a 4-to-2 encoder and a 1:2 demultiplexer. It is really rare for one molecule to mimic so many logic operations.
\end{abstract}

\section{Introduction}

To develop an effective detection method for anions has become an important subject due to the crucial role of anions in biological, chemical and environmental systems, especially in the fields of biological metabolites, food additives, drugs, agricultural fertilizers, and waste liquids. ${ }^{1-3}$ Among anions, cyanide, fluoride and phosphate can cause huge damage to the environment, such as the production of toxic waste, water contamination and eutrophication, and nerve gases. ${ }^{4-6}$ Consequently, there is demand for sensitive, selective and easy-to-manipulate methods for detecting these three kinds of toxic anions. However, compared to the cation binding and sensing, anion detection is more challenging for their large size, variable shape, weak electrostatic interactions, and strong solvation effects. ${ }^{7}$ In addition, various non-covalent interactions, such as hydrogen bonding, hydrophobicity, and coordination to metal center, would impose a huge effect on the recognition of anions. ${ }^{\mathbf{8} 9}$ As a kind of simple

${ }^{a}$ College of Materials Science and Engineering, Fujian Key Laboratory of Polymer Materials, Fujian Normal University, Fuzhou 350007, China

${ }^{b}$ College of Chemistry and Chemical Engineering, Fujian Normal University, Fuzhou 350007, China.E-mail: zhlin@fjnu.edu.cn; lingqd@fjnu.edu.cn

$\dagger$ Electronic supplementary information (ESI) available: Absorption and emission spectra, photophysical and TD-DFT calculation data, titration curves, as well as Job's plot. See DOI: 10.1039/c6ra28367e detection method of anions, fluorimetric probing and sensing have attracted wide interest of researchers for excellent sensitivity, good specificity, a large linear range of analysis, and ease of handling. ${ }^{10}$ The interactions of anions with fluorescent sensors often introduce new optical transitions or excited states and lead to changes in the photophysical properties (such as the absorption spectra, emission intensity, or lifetime, etc.), which can then act as an indicator of anion recognition. ${ }^{\mathbf{1 1 , 1 2}}$

Molecular logic gates are an interdisciplinary research area that could make integrated circuits smaller and more efficient, compared with traditional silicon-based circuits. ${ }^{\mathbf{1 3 , 1 4}}$ As a result, mimicking digital logic operations of the real world in the molecular level has attracted increasing interest. ${ }^{\mathbf{1 5}}$ Since the first AND molecular logic gate was reported by De Silva, ${ }^{16}$ all kinds of molecular systems mimicking the functions of logic devices, even complicated computing operation (such as multiplexer, demultiplexer, adder, subtractor, encoder and decoder etc.), have been developed in the last two decades. ${ }^{\mathbf{1 3 , 1 7 - 1 9}}$ Stimuli-responsive fluorescent materials display great potential in the field of molecular computing for their excellent sensitivity, good specificity, a large linear range of analysis, and ease of handling. ${ }^{17,20-22}$ If the conversation process from external stimuli (cation/anion, acid/base, redox, DNA, light, heat, chemicals and electricity) to the response in the photophysical properties complies with the binary Boolean logic rules, the function of logic devices can be 
realized by employing the stimulus and response as input and output, individually. ${ }^{23-29}$

Anion recognition of fluorescent sensors is frequently used to construct the molecular logic system for the significant availability of anion interaction..$^{2,26,30-32}$ However, one sensor molecule always responds to anions in one channel, which means that selective detecting multiple anions need different sensors, and that complex molecular design, incorporating other functional groups into the sensor, is necessary to meet the need of logic operation. ${ }^{33,34}$ If one molecule can selectively sense multiple anions (such as $\mathrm{F}^{-}, \mathrm{CN}^{-}$and $\mathrm{H}_{2} \mathrm{PO}_{4}{ }^{-}$etc.) in different signaling channels, it is desirable for both selective anion recognition and molecular logic computing.

Indolylmaleimide derivatives have been successfully applied in organic light-emitting diode as electroluminescent materials, due to their strong luminescence in both solid and solution. ${ }^{35-38}$ Recently, we expand the application of the 3,4-diarylmaleimide derivatives with $\mathrm{N}-\mathrm{H}$ functional groups (indole, pyrrole or amide) to sense anions. ${ }^{39,40}$ Maleimide, as an electron-deficient group, can help enhance the acidity and $\mathrm{H}$-bond binding ability of $\mathrm{N}-\mathrm{H}$ group in the indole rings. As a result, the optical properties of indolylmaleimides are sensitive to $\mathrm{NH}$-anion interactions, thus suitable for both colorimetric and fluorimetric detections of anions. It is necessary to investigate systematically the effect of different $N$-substituent of maleimide, solvents and molecular planarity on the sensing capacity of indolylmaleimides to obtain one compound sensing multiple anions in different ways. To this end, in this paper, a series of 3,4-bisindolylmaleimide dyes (IMs, Scheme 1) with $\mathrm{N}-\mathrm{H}$ groups were designed and synthesized to detect anions in different solvents. It is found that medium polarity and molecular planarity have an important influence on the selectivity of anions recognition. Through changing the solvent from ACN, DCM to THF, IM-PFB could selectively detect $\mathrm{F}^{-}, \mathrm{CN}^{-}$and $\mathrm{H}_{2} \mathrm{PO}_{4}{ }^{-}$. According to the results, three kinds of decoders (1-to-2, 2-to-3 and 2-to-4), a 4-to-

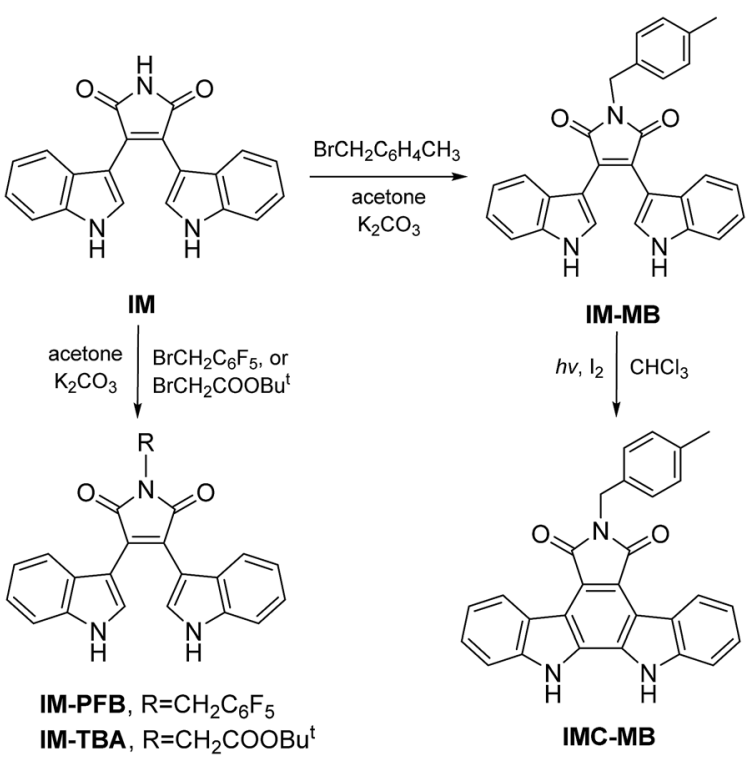

Scheme 1 Structure and synthesis of bisindolylmaleimide derivatives.
2 encoder and a 1:2 demultiplexer have been constructed by IM-PFB.

\section{Experimental section}

\section{General}

Electronic spray ion (ESI) mass spectra were recorded on a Bruker micrOTOF II spectrometer. NMR spectra were measured in $\mathrm{CDCl}_{3}$, $d$-acetone or $d$-DMSO on a Bruker Ascend 400 FT-NMR spectrometer; ${ }^{1} \mathrm{H}$ and ${ }^{13} \mathrm{C}$ chemical shifts were quoted relative to the internal standard tetramethylsilane. UV-vis spectra were obtained on a Shimadzu UV-2600 spectrophotometer. The PL spectra were probed on a Shimadzu RF-5301PC fluorescence spectrophotometer. All photographs were recorded on a Canon Powershot G7 digital camera under daylight or UV light (365 nm) lamp. The fluorescence quantum yields were determined in $\mathrm{CH}_{3} \mathrm{CN}$ solutions at $293 \mathrm{~K}$ against Nile red as a reference $\left(\Phi_{\mathrm{p}}=0.78\right){ }^{41}$

All of the reagents and solvents used were obtained from commercial suppliers and were used without further purification unless otherwise noted. Thin layer chromatography was performed on G254 plates of Qingdao Haiyang Chemical. Column chromatography was performed on Sorbent Technologies brand silica gel (40-63 mm, Standard grade).

\section{Synthetic procedure of IM-PFB, IM-TBA and IM-MB}

To a solution of IM $(1.5 \mathrm{~g}, 4.6 \mathrm{mmol})$ in acetone $(12 \mathrm{~mL})$ under a nitrogen atmosphere were added bromide $(4.6 \mathrm{mmol})$ and $\mathrm{K}_{2} \mathrm{CO}_{3}(0.76 \mathrm{~g}, 5.5 \mathrm{mmol})$. The mixture was allowed to be stirred for $12 \mathrm{~h}$ at ambient temperature. The mixture was successively extracted with ethyl acetate and washed with water and brine. It was then dehydrated over anhydrous $\mathrm{MgSO}_{4}$. After removing the solvent, the crude product was purified by column chromatography with ethyl acetate/petroleum ether $(1: 5)$ as the eluant, affording pure dark red solids in $41-77 \%$ yield.

Physical data for IM-PFB (68\%). ${ }^{1} \mathrm{H}$ NMR $\left(\mathrm{CDCl}_{3}, 400 \mathrm{MHz}\right)$ : $\delta 8.58(\mathrm{~s}, 2 \mathrm{H}), 7.75(\mathrm{~d}, 2 \mathrm{H}), 7.32(\mathrm{~d}, 2 \mathrm{H}), 7.07(\mathrm{t}, 2 \mathrm{H}), 6.94(\mathrm{~d}, 2 \mathrm{H})$, $6.75(\mathrm{t}, 2 \mathrm{H}), 4.97(\mathrm{~s}, 2 \mathrm{H}) .{ }^{13} \mathrm{C}$ NMR $(d$-DMSO, $100 \mathrm{MHz}): \delta 171.03$, 146.69, 144.18, 138.54, 136.46, 129.87, 127.33, 125.72, 122.16, $121.41,119.88,112.26,111.00,105.96,30.02$. HRMS (ESI) $\mathrm{m} / \mathrm{z}$ $[\mathrm{M}]^{+}$calcd 507.1006, found 507.1000.

Physical data for IM-TBA (41\%). ${ }^{1} \mathrm{H}$ NMR ( $d$-acetone, 400 MHz): $\delta 10.80(\mathrm{~s}, 2 \mathrm{H}), 7.83(\mathrm{~d}, 2 \mathrm{H}), 7.47(\mathrm{~d}, 2 \mathrm{H}), 6.97(\mathrm{t}, 2 \mathrm{H}), 6.93$ $(\mathrm{d}, 2 \mathrm{H}), 6.62-6.66(\mathrm{~m}, 2 \mathrm{H}), 4.30(\mathrm{~s}, 2 \mathrm{H}), 1.40(\mathrm{~s}, 9 \mathrm{H}) .{ }^{13} \mathrm{C} \mathrm{NMR}(d-$ acetone, $100 \mathrm{MHz}): \delta 171.27,166.92,136.43,129.67,127.65$, $125.88,121.93$, 121.44, 120.00, 111.07, 106.50, 81.43, 39.91, 27.26. HRMS (ESI) $m / z[\mathrm{M}]^{+}$calcd 441.1689, found 441.1683.

Physical data for IM-MB (77\%). ${ }^{1} \mathrm{H}$ NMR $\left(\mathrm{CDCl}_{3}, 400 \mathrm{MHz}\right)$ : $\delta 8.47(\mathrm{~s}, 2 \mathrm{H}), 7.76(\mathrm{~d}, 2 \mathrm{H}), 7.38-7.31(\mathrm{~m}, 4 \mathrm{H}), 7.06-7.15(\mathrm{~m}, 4 \mathrm{H})$, $6.95(\mathrm{~d}, 2 \mathrm{H}), 6.74(\mathrm{t}, 2 \mathrm{H}), 4.81(\mathrm{~s}, 2 \mathrm{H}), 2.31(\mathrm{~s}, 3 \mathrm{H}) .{ }^{13} \mathrm{C}$ NMR $(d-$ DMSO, $100 \mathrm{MHz}): \delta 171.92,137.01,136.45,134.78,129.80,129.60$, 128.00 , 127.34, 125.81, 122.14, 121.40, 119.86, 112.25, 106.05, 41.33, 21.13. HRMS (ESI) $m / z[\mathbf{M}]^{+}$calcd 431.1634, found 431.1628.

\section{Synthetic procedure of compound IMC-MB}

To a red solution of IM-MB $(0.67 \mathrm{~g}, 1.56 \mathrm{mmol})$ in $500 \mathrm{~mL}$ of benzene was added iodine $(0.04 \mathrm{~g}(0.156 \mathrm{mmol}))$. The reaction 
mixture continuously bubbled with air was irradiated with a medium pressure mercury lamp for $8 \mathrm{~h}$. The crude products were diluted with ethyl acetate, extracted with saturated $\mathrm{Na}_{2} \mathrm{~S}_{2} \mathrm{O}_{4}$, rinsed with brine, dried over $\mathrm{Na}_{2} \mathrm{SO}_{4}$, filtered, and concentrated in vacuum. Purification by flash chromatography with ethyl acetate/petroleum ether $(1: 4)$ as the eluant yielded $0.8366 \mathrm{~g}(57 \%)$ of IMC-MB as a yellow solid.

Physical data for IMC-MB (57\%). ${ }^{1} \mathrm{H} \mathrm{NMR}\left(\mathrm{CDCl}_{3}, 400 \mathrm{MHz}\right)$ : $\delta 9.31$ (s, 2H), 9.04 (d, 2H), 7.51-7.37 (m, 8H), 7.15 (d, 2H), 4.87 $(\mathrm{s}, 2 \mathrm{H}), 2.30(\mathrm{~s}, 3 \mathrm{H}) .{ }^{13} \mathrm{C}$ NMR (d-DMSO, $\left.100 \mathrm{MHz}\right): \delta 169.99$, 140.84, 136.92, 135.04, 130.09, 129.55, 128.00, 127.39, 124.66, 121.90, 120.76, 119.01, 116.21, 112.56, 40.90, 21.11. HRMS (ESI) $\mathrm{m} / \mathrm{z}[\mathrm{M}]^{+}$calcd 429.1477, found 429.1471.

\section{Evaluation of sensing capability}

The evaluation of anion-sensing capability of IM-PFB, IM-TBA, IM-MB and IMC-MB was performed in acetonitrile (ACN), dichloromethane (DCM) and tetrahydrofuran (THF) by the addition of the tetrabutylammonium salt of various anions such as $\mathrm{NO}_{3}{ }^{-}, \mathrm{Br}^{-}, \mathrm{HSO}_{4}{ }^{-}, \mathrm{Cl}^{-}, \mathrm{OAc}^{-}, \mathrm{CN}^{-}, \mathrm{H}_{2} \mathrm{PO}_{4}{ }^{-}, \mathrm{F}^{-}$and $\mathrm{I}^{-}$. These solvents are analytic reagent and used without drying. All of evaluation experiments including optical spectra collecting were performed under air atmosphere. Freshly prepared samples in $1 \mathrm{~cm}$ quartz cells were used for all absorption and emission measurements.

\section{Results and discussion}

\section{Synthesis and photophysical properties}

The synthetic route of four bisindolylmaleimide dyes (IMs) was presented in Scheme 1. IM-PFB, IM-TBA and IM-MB were obtained readily from 3,4-bis(3-indolyl)-maleimide ${ }^{42}$ through an alkylation under alkaline condition. IM-MB went through an oxidative cyclization catalyzed by $\mathrm{I}_{2}$ and UV light to give IMCMB. All of the resulting compounds were characterized by ${ }^{1} \mathrm{HNMR},{ }^{13} \mathrm{CNMR}$ and high resolution mass spectra.

In THF solution, IM-PFB, IM-TBA and IM-MB exhibit similar absorption and emission properties with their normalized spectra shown in Fig. 1. It indicates that the spectral properties of these compounds in solution are not influenced much by changing the $\mathrm{N}$-substituent on maleimide. There are three major absorption bands in the range of 250-550 $\mathrm{nm}$ regions of their UV-vis spectra. The band before $320 \mathrm{~nm}$ is assigned to a $\pi-$ $\pi^{*}$ transition localized mainly on the indole group. ${ }^{43}$ The low energy bands after $320 \mathrm{~nm}$ are derived from the transitions involving both indole and maleimide units, because neither of them alone exhibits an absorption band after $325 \mathrm{~nm}$. The broad featureless band at about $457 \mathrm{~nm}$ displays an intramolecular charge-transfer (ICT) character, which corresponds to an electron transition from indole to maleimide. ${ }^{44}$ Charge recombination from the excited state gives a red emission at 597-602 nm showing an apparent solvent shift (Fig. S1†), with $30-34 \%$ of the quantum efficiency (Table S1 $\dagger$ ).

Compared to IM-PFB, IM-TBA and IM-MB, IMC-MB shows a completely different absorption and emission spectra in solution, due to its flat structure (Fig. 1). There are two major

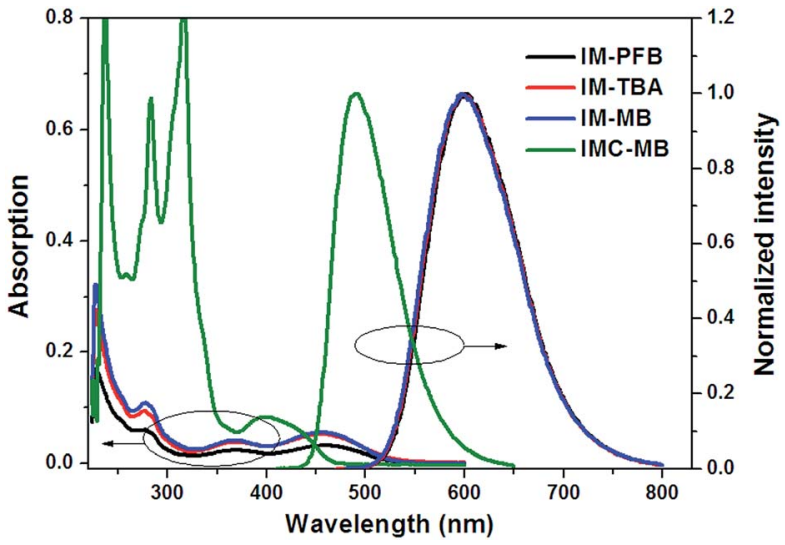

Fig. 1 Absorption and normalized emission spectra of sensors in THF $(50 \mu \mathrm{M})$.

absorption bands in the absorption spectrum of IMC-BM after $260 \mathrm{~nm}$, one in the UV region around $317 \mathrm{~nm}$, with shoulder at $283 \mathrm{~nm}$, and the other one around $399 \mathrm{~nm}$ in the visible region, which can be assigned to $\pi-\pi^{*}$ transitions from the $S_{0}$ to the $S_{2}$ and $S_{1}$ states, respectively. When irradiated at $399 \mathrm{~nm}$, IMC-MB exhibits intense green luminescent at $490 \mathrm{~nm}$ with a higher quantum yield of $39 \%$ as compared to IM-MB (Table S1 $\dagger$ ).

\section{Qualitative evaluation}

Effect of substituent. The qualitative evaluation of anion sensing capability of IM-MB, IM-TBA and IM-PFB was performed in acetonitrile by the addition of the tetrabutylammonium salt of various anions such as $\mathrm{NO}_{3}{ }^{-}, \mathrm{Br}^{-}, \mathrm{HSO}_{4}{ }^{-}, \mathrm{Cl}^{-}$, $\mathrm{OAc}^{-}, \mathrm{CN}^{-}, \mathrm{H}_{2} \mathrm{PO}_{4}^{-}, \mathrm{F}^{-}$and $\mathrm{I}^{-}$(Fig. 2, S2 and S3†). As noted in Fig. 2a, IM-MB exhibits highly selective sensing for $\mathrm{F}^{-}$with fluorescence quenching and color change from yellow to purple. The color change of IM-MB upon $\mathrm{F}^{-}$is originated from the disappearance of the absorption band around $455 \mathrm{~nm}$ along with two new peaks at $565 \mathrm{~nm}$ and $323 \mathrm{~nm}$ (Fig. 2b). The ratio of absorption intensities at $565 \mathrm{~nm}$ and $455 \mathrm{~nm}\left(I_{565} / I_{455}\right)$ presents a 265 fold enhancement, from 0.02 without $\mathrm{F}^{-}$to 5.3 with $\mathrm{F}^{-}(20$ eq.). When IM-TBA and IM-PFB was added by $\mathrm{F}^{-}$, similar alteration in absorption spectra was found. The ratio of $I_{565} / I_{455}$ increases 1876 fold for IM-TBA and 2671 fold for IM-MB with $\mathrm{F}^{-}$ (100 eq.), as shown in Fig. S2a and S3b. $\dagger$ In addition, the peak around $606 \mathrm{~nm}$ in the emission spectra almost disappears after the addition of $\mathrm{F}^{-}$(Fig. 2c, S2b and S3c $\dagger$ ). Consequently, IMPFB, IM-TBA and IM-MB, as a kind of colorimetric and fluorescent sensors, can efficiently and selectively detect fluoride in ACN solution. The above results also indicate that $N$-substituents of maleimide are insignificant for their selective recognition of anions.

Effect of solvent. As mentioned before, the 3,4-bisindolylmaleimide dyes are of ICT character, resulting in their emission affected by the polarity of solvents. Would the polarity of solvents impose effect on their sensing for anions? To answer this question, the qualitative evaluation of anion recognition of these 3,4-bisindolylmaleimides IMs was further proceeded in DCM and THF solvents. In DCM (Fig. 3a, c and S4†), IM-TBA 
a)

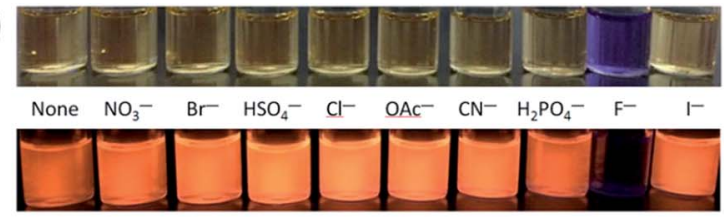

b)

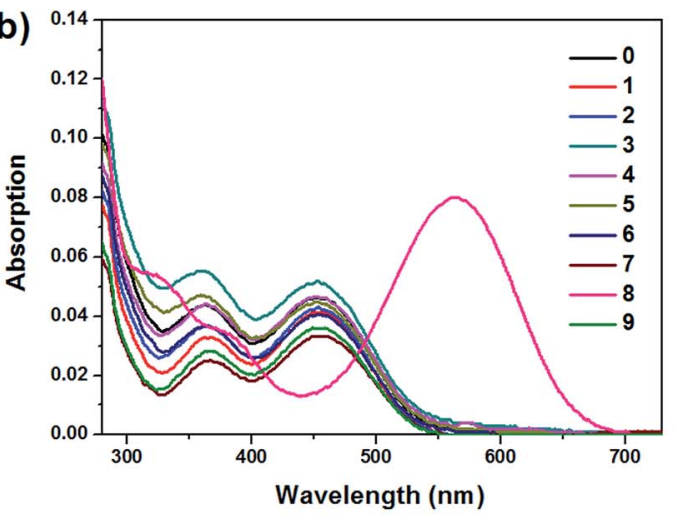

c)

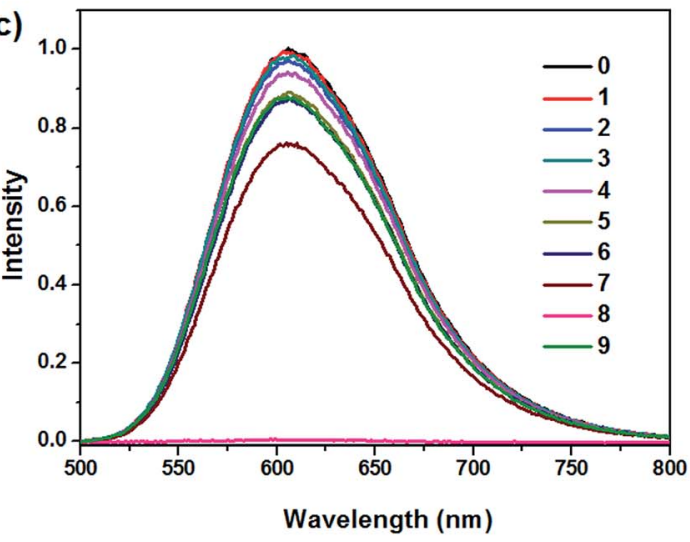

Fig. 2 (a) Photograph of $I M-M B(50 \mu M$ in $A C N)$ in the presence of various anions (20 eq.) under daylight (top) and UV light (bottom). Absorption (b) and emission (c) spectra of IM-MB (50 $\mu \mathrm{M}$ in ACN) in the presence of anions (20 eq.). Anions arrangement: 0-none, 1- $\mathrm{NO}_{3}{ }^{-}, 2-$ $\mathrm{Br}^{-}, 3-\mathrm{HSO}_{4}^{-}, 4-\mathrm{Cl}^{-}, 5-\mathrm{OAc}^{-}, 6-\mathrm{CN}^{-}, 7-\mathrm{H}_{2} \mathrm{PO}_{4}^{-}, 8-\mathrm{F}^{-}, 9-1^{-}$.

and IM-PFB show similar sensing ability as the previous reported bisindolylmaleimide. ${ }^{40}$ That is, only $\mathrm{F}^{-}$induces the color of IM-TBA and IM-PFB change from faint yellow into lavender. However, both $\mathrm{H}_{2} \mathrm{PO}_{4}^{-}$and $\mathrm{F}^{-}$cause their fluorescent quenching in DCM. Differently from the change of absorption spectra of IM-PFB and IM-TBA upon $\mathrm{F}^{-}$in ACN, their absorption bands around $370 \mathrm{~nm}$ and $460 \mathrm{~nm}$ show a bathochromic shift in DCM upon the addition of 100 eq. of $\mathrm{H}_{2} \mathrm{PO}_{4}{ }^{-}$and $\mathrm{F}^{-}$. For example, the maximum absorption wavelength of IM-TBA shifts from $456 \mathrm{~nm}$ to $473 \mathrm{~nm}$ upon $\mathrm{H}_{2} \mathrm{PO}_{4}{ }^{-}$and to $551 \mathrm{~nm}$ upon $\mathrm{F}^{-}$ (Fig. 3c). The emission intensity of IM-TBA in DCM at $604 \mathrm{~nm}$ is quenched $86 \%$ and $94 \%$ by $\mathrm{H}_{2} \mathrm{PO}_{4}{ }^{-}$and $\mathrm{F}^{-}$, respectively (Fig. $3 \mathrm{c}$ inset).

In THF (Fig. 3b, d and S5†), obvious response to the optical properties of IM-TBA and IM-PFB is found after addition of $\mathrm{CN}^{-}$ and $\mathrm{F}^{-}$. Their change in absorption and emission spectra is similar as that induced by $\mathrm{F}^{-}$in ACN. For instance, the absorption band of IM-TBA around $460 \mathrm{~nm}$ in THF disappears, accompanied by two new peaks at $570 \mathrm{~nm}$ and $323 \mathrm{~nm}$ in the a)

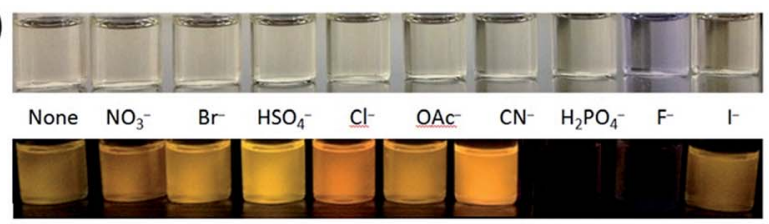

b)

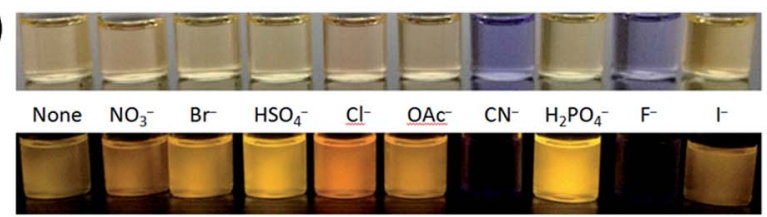

c)

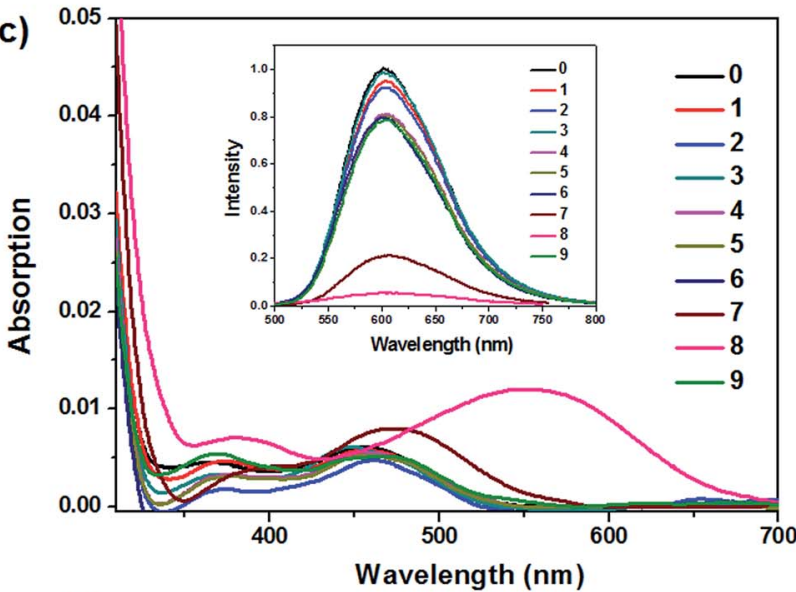

d)

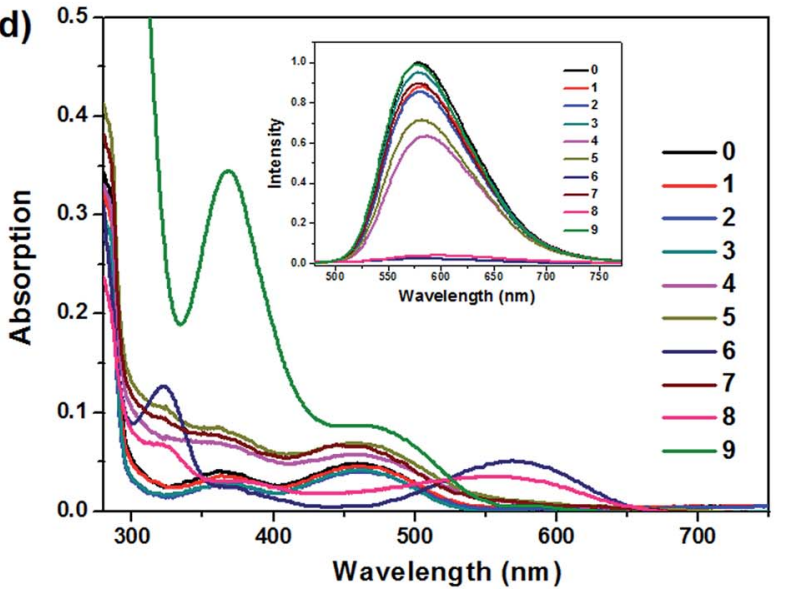

Fig. 3 Photograph of IM-TBA $(50 \mu \mathrm{M})$ in DCM (a) and THF (b) upon various anions (100 eq.) under daylight (top) and UV light (bottom). Absorption and emission (inset) spectra of IM-TBA (50 $\mu \mathrm{M})$ in DCM (c) and THF (d) upon anions (100 eq.). Anions arrangement: 0-none, 1$\mathrm{NO}_{3}{ }^{-}, 2-\mathrm{Br}^{-}, 3-\mathrm{HSO}_{4}{ }^{-}, 4-\mathrm{Cl}^{-}, 5-\mathrm{OAc}^{-}, 6-\mathrm{CN}^{-}, 7-\mathrm{H}_{2} \mathrm{PO}_{4}^{-}, 8-\mathrm{F}^{-}, 9-\mathrm{I}^{-}$.

presence of $\mathrm{CN}^{-}$(Fig. 3d). Although the degree of fluorescent quenching of IM-TBA and IM-PFB by $\mathrm{CN}^{-}$and $\mathrm{F}^{-}$is considerable, their colorimetric sensing sensitivity $\left(I_{570} / I_{460}\right)$ for $\mathrm{CN}^{-}$is much bigger than that for $\mathrm{F}^{-}$.

Effect of cyclization. IMC-MB obtained by the cyclization of IM-MB was employed to investigate the effect of the planarity of 3,4-bisindolylmaleimide on sensing for anions (Fig. S6-S8†). As mentioned above, IM-MB showed an effectively colorimetric and fluorescent recognition only for $\mathrm{F}^{-}$in ACN. However, the difference of absorption spectra of IMC-BM in the presence of 
various anions is inconspicuous in $\mathrm{ACN}$ (Fig. $\mathrm{S} 6 \mathrm{~b} \dagger$ ). There are four kinds of anions $\left(\mathrm{OAc}^{-}, \mathrm{CN}^{-}, \mathrm{H}_{2} \mathrm{PO}_{4}^{-}\right.$and $\mathrm{F}^{-}$) causing an impressive decline in emission intensity of IMC-BM (Fig. S6c $\dagger$ ). In DCM and THF solvent, IMC-BM also exhibited poorer selectivity of anion sensing than IM-PFB and IM-TBA (Fig. S7 and $\mathrm{S} 8{ }^{\dagger}$ ), which should be attributed to its weak ICT character and acidity of $\mathrm{NH}$ in indole units, as well as the fixed location of two $\mathrm{NH}$ receptor for anion recognition. Thus, the relative orientation between the two $\mathrm{NH}$ groups of IMs should be important in selective sensing for anions.

Selective recognition of anions. It can be found that the solvent environment of these dyes play a more important role in the selective recognition of anions than substituent and molecular planarity. They showed different spectra response to anions in different solvents. Taking IM-TBA for example, based on above the qualitative evaluation (Fig. $\mathrm{S} 2, \dagger 3 \mathrm{c}$ and $\mathrm{d}$ ), the intensity changes of its absorption and emission spectra in different solvents are summarized in Fig. 4. If the ordinate value of absorption (Fig. 4a, c and e) more than 3 or emission (Fig. 4b, $\mathrm{d}$ and $\mathrm{f}$ ) less than 0.5 , are considered response to anions, the response of IM-TBA to anions in different solvents can be summarized in Table 1. In THF solvent, the response on both the absorption and emission of IM-TBA is caused by $\mathrm{CN}^{-}$, while only response to its emission induced by $\mathrm{F}^{-}$. In DCM solvent, response to emission, not to absorption of IM-TBA was found in the presence of $\mathrm{H}_{2} \mathrm{PO}_{4}{ }^{-}$. Only $\mathrm{F}^{-}$can lead to both of absorption and emission response of IM-TBA in ACN and DCM solvents. Consequently, IM-TBA can selectively recognize $\mathrm{CN}^{-}$in THF,
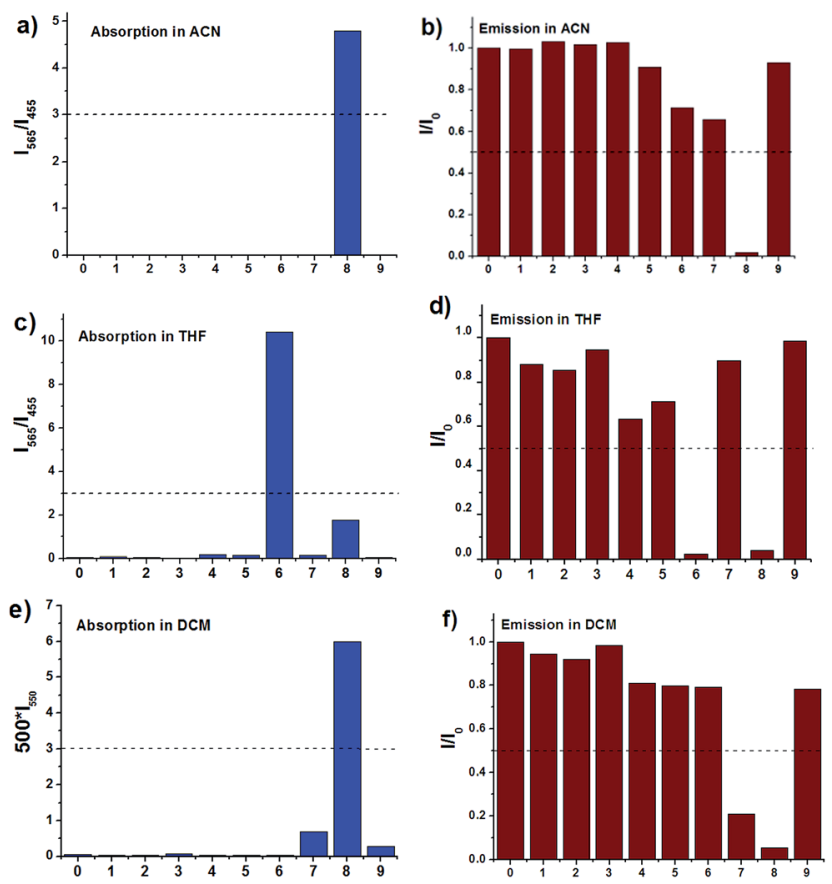

Fig. 4 The ratio of absorption intensity $/_{565} / /_{455}$ (a and c) of IM-TBA in $A C N$ and THF upon various anions (100 eq.). The absorption intensity at $550 \mathrm{~nm}$ (e) of IM-TBA in DCM upon various anions (100 eq.). The relative emission intensity ( $b, d$ and $f$ ) of IM-TBA at $609 \mathrm{~nm}$ in the presence of anions (100 eq.). Anions arrangement: 0 -none, $1-\mathrm{NO}_{3}{ }^{-}$, 2- $\mathrm{Br}^{-}, 3-\mathrm{HSO}_{4}{ }^{-}, 4-\mathrm{Cl}^{-}, 5-\mathrm{OAc}^{-}, 6-\mathrm{CN}^{-}, 7-\mathrm{H}_{2} \mathrm{PO}_{4}^{-}, 8-\mathrm{F}^{-}, 9-\mathrm{I}^{-}$.
Table 1 Response of IM-TBA to anions in different solvents ${ }^{a}$

\begin{tabular}{|c|c|c|c|c|c|c|}
\hline & \multicolumn{2}{|c|}{$\mathrm{ACN}$} & \multicolumn{2}{|c|}{ DCM } & \multicolumn{2}{|c|}{$\mathrm{THF}^{a}$} \\
\hline & uv & $\mathrm{pl}$ & uv & $\mathrm{pl}$ & uv & $\mathrm{Pl}$ \\
\hline $\mathrm{CN}^{-}$ & - & - & - & - & $\mathrm{R}$ & $\mathrm{R}$ \\
\hline $\mathrm{H}_{2} \mathrm{PO}_{4}^{-}$ & - & - & - & $\mathrm{R}$ & - & - \\
\hline $\mathrm{F}^{-}$ & $\mathrm{R}$ & $\mathrm{R}$ & $\mathrm{R}$ & $\mathrm{R}$ & - & $\mathrm{R}$ \\
\hline Others & - & - & - & - & - & - \\
\hline
\end{tabular}

and $\mathrm{H}_{2} \mathrm{PO}_{4}{ }^{-}$in DCM, and $\mathrm{F}^{-}$in ACN through monitoring its optical performance of the absorption and emission.

\section{Spectra titration in different solvent}

The titration curves of fluoride for IM-PFB and IM-MB in ACN are similar and shown in Fig. 5a, b and S9, $\dagger$ respectively. Their absorption peaks at $455 \mathrm{~nm}$ and $367 \mathrm{~nm}$ decrease upon addition of fluoride along with the increasing of $564 \mathrm{~nm}$ and $325 \mathrm{~nm}$ bands. Two distinct isosbestic points can be found at 493 and $343 \mathrm{~nm}$, indicating the formation of a stable new species. In their emission spectra in ACN, the intensity of $609 \mathrm{~nm}$ band decreases gradually with the increase in the concentration of $\mathrm{F}^{-}$, accompanied by a small red-shift of the maximum wavelength. When the amount of $\mathrm{F}^{-}$reaches 10 eq., the emission peaks shifted to $641 \mathrm{~nm}$ almost disappears in ACN.

The spectra response of IM-PFB to $\mathrm{F}^{-}$and $\mathrm{H}_{2} \mathrm{PO}_{4}{ }^{-}$in DCM differs from that in ACN (Fig. 5c, d and S10†). Taking $\mathrm{F}^{-}$for example, the long wavelength absorption at 462 gradually and bathochromically shifts to $520 \mathrm{~nm}$ with the amount of $\mathrm{F}^{-}$ increasing from 0 to 60 eq. Concurrently, the emission intensity at $604 \mathrm{~nm}$ decreases to $3 \%$ without apparent wavelength shift. When the amount of $\mathrm{F}^{-}$further increased to 80 eq., the absorption and emission spectra keep untouched. The change is similar as that of the reported bisindolylmaleimide titrated by anions in DCM, which indicate IM-PFB should sense $\mathrm{F}^{-}$and $\mathrm{H}_{2} \mathrm{PO}_{4}{ }^{-}$through hydrogen bonding interaction. ${ }^{40}$
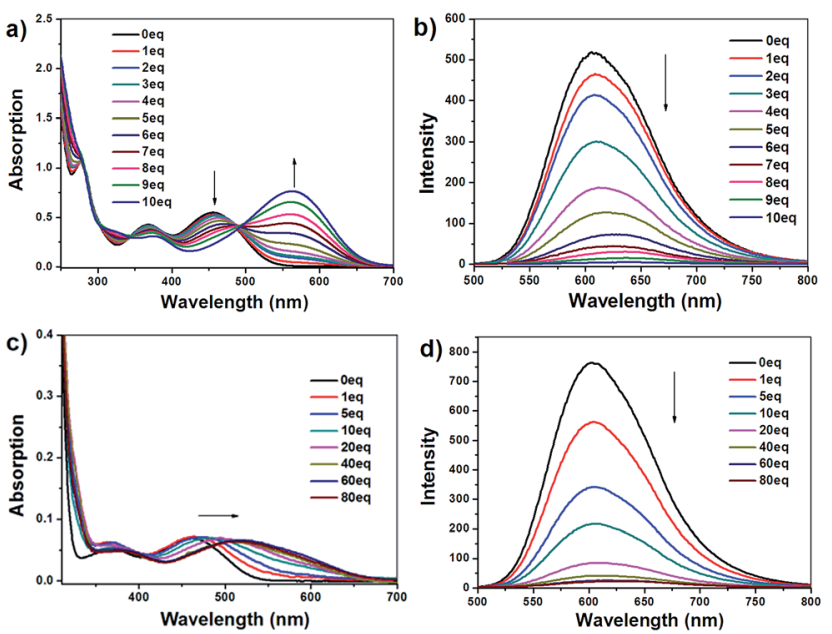

Fig. 5 Absorption ( $a$ and $c$ ) and emission ( $b$ and $d$ ) spectra of IM-PFB (5 $\mu \mathrm{M})$ in $\mathrm{ACN}\left(\mathrm{a}\right.$ and $\mathrm{b}$ ) and $\mathrm{DCM}$ ( $\mathrm{c}$ and $\mathrm{d}$ ) upon titration of $\mathrm{F}^{-}$. 
In THF solvent, IM-PFB can selectively detect $\mathrm{CN}^{-}$and $\mathrm{F}^{-}$ from other anions with the titration curves displayed in Fig. S11 and $\mathrm{S} 12 . \dagger$ Upon titration of $\mathrm{CN}^{-}$and $\mathrm{F}^{-}$in THF, the absorption spectra of IM-PFB exhibit similar change trend as that of titration of $\mathrm{F}^{-}$in ACN. That is, absorption band at $466 \mathrm{~nm}$ reduces with concomitant growth of two new bands at $575 \mathrm{~nm}$ and $321 \mathrm{~nm}$. However, the quenching degree of emission in THF is less than that in ACN. For instance, 10 eq. of $\mathrm{F}^{-}$can quench the fluorescence of IM-PFB and IM-MB in ACN to $9 \%$ and $1 \%$, respectively. While 10 eq. of $\mathrm{F}^{-}$and $\mathrm{CN}^{-}$can only cause the emission intensity of IM-PFB in THF decrease to $44 \%$ and $14 \%$, respectively.

\section{Sensing mechanism}

IM-PFB was chosen as a model to analyze the sensing mechanism of anions. As mentioned above, IM-PFB should sense $\mathrm{F}^{-}$ and $\mathrm{H}_{2} \mathrm{PO}_{4}{ }^{-}$in DCM through hydrogen bonding interaction because there is no distinct isosbestic points found in the titration absorption spectra. ${ }^{40}$ The titration spectra of anions in ACN and THF indicate that IM-PFB possibly detects $\mathrm{F}^{-}$and $\mathrm{CN}^{-}$ in a different way for the formation of new species. There are mainly two kinds of sensing mechanism of sensors based on the $\mathrm{NH}$ group as recognition sites: hydrogen bonding and deprotonation. ${ }^{\mathbf{4 0 , 4 5 , 4 6}}$ Consequently, it can be supposed that IMPFB in ACN and THF could be turned into anion by $\mathrm{F}^{-}$or $\mathrm{CN}^{-}$ through a deprotonation reaction. To verify the supposition, the reactions of IM-PFB with a strong base $\mathrm{N}(\mathrm{Et})_{4} \mathrm{OH}$ in different solvents, as well as theory calculations were employed to analyze the optical properties of IM-PFB and its anion. Fig. S13† shows the absorption and fluorescence spectra of IM-PFB $(50 \mu \mathrm{M})$ in DCM upon the addition of different amounts of $\mathrm{N}(\mathrm{Et})_{4} \mathrm{OH}$. With the increasing concentration of $\mathrm{OH}^{-}$, the absorption peak at $457 \mathrm{~nm}$ gradually disappears, accompanied with the appearance of a new absorption peak at around $570 \mathrm{~nm}$ and the reduction of emission intensity. Similar situation was found in solvents THF and ACN with the new absorption peak appeared at $576 \mathrm{~nm}$ and $566 \mathrm{~nm}$, individually (Fig. S14 and S15†). In ACN, with the continued increase of $\mathrm{OH}^{-}$amount, another new absorption peak at $648 \mathrm{~nm}$ replaces the peak at $566 \mathrm{~nm}$. The first new peak at about $570 \mathrm{~nm}$ and the second new peak at around $650 \mathrm{~nm}$ should be assigned to monovalent and divalent anion of IM-PFB (IM-PFB ${ }^{-}$and $\mathrm{IMPFB}^{2-}$ ), corresponding to the deprotonation of one hydrogen atom and two hydrogen atoms, individually. It would be confirmed by the following results of theory calculation.

Molecular optimization and optical transitions of IM-PFB and its anions were performed by the density function (DFT) and time-dependent density function (TD-DFT) theory with B3LYP using 6-31G(d) basis sets. In the optimized geometry of IM-PFB (Fig. 6b), the two indole rings are oriented anti to each other, with two $\mathrm{N}$ atoms pointing outward. Its electron density distributions of HOMO and LUMO can be depicted in Fig. 6a. As expected the electron density in the HOMO of IM-PFB is mainly populated on two symmetrically equivalent indolyl moieties, while the LUMO is localized on the central maleimide moiety. A charge separation is expected to be produced when
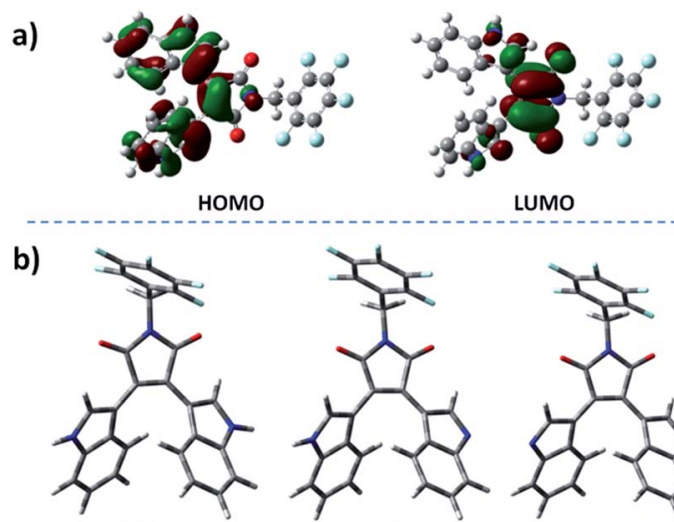

IM-PFB

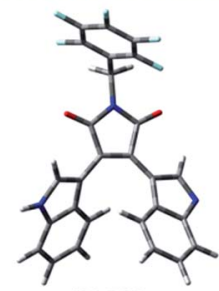

IM-PFB-
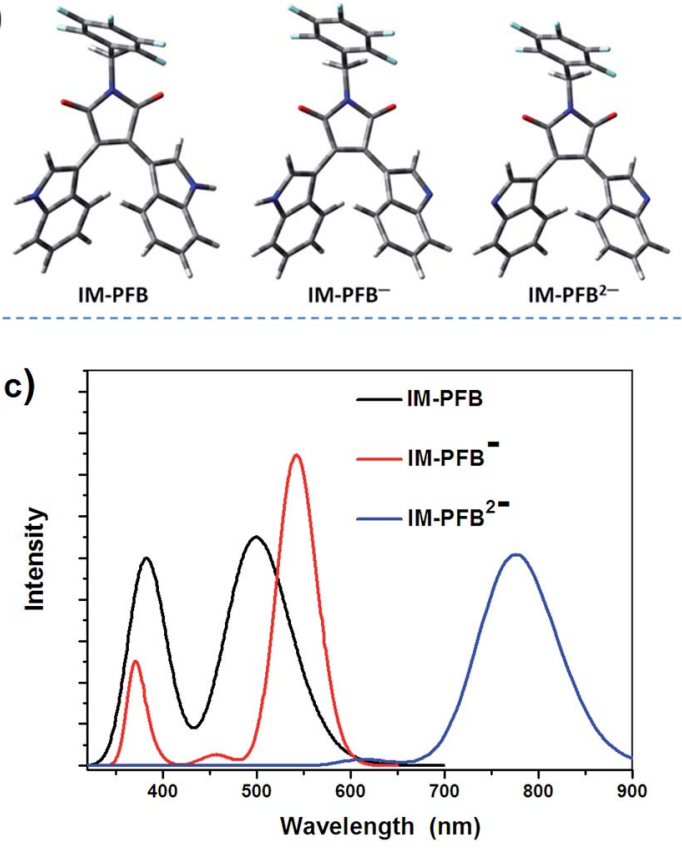

Fig. 6 Electron density distributions of $\mathrm{HOMO}$ and LUMO of IM-PFB (a). Optimized geometry (b) and stimulated absorption spectra (c) of IM-PFB and its anion.

an electron is promoted from the HOMO to the LUMO, which make the $\mathrm{NH}$ group of indole rings easy to form a hydrogen bond or be deprotonated by a base. From the optimized structure of $\mathrm{IM}^{-\mathrm{PFB}^{-}}$and $\mathrm{IMPFB}^{2-}$ (Fig. 6b), deprotonation does not change much the geometries of indole rings. The absorption spectra of IM-PFB and its anions were simulated by TD-DFT calculations and showed in Fig. $6 \mathrm{c}$ with the calculated data summarized in Table S2. $\dagger$ The wavelength correlates remarkably well with the experimental results of IM-PFB treated with $\mathrm{OH}^{-}$(Fig. S13-S15 $\dagger$ ). For example, the calculated main absorption wavelengths of IM-PFB, IM-PFB ${ }^{-}$and $\mathrm{IMPFB}^{2-}$ are $499 \mathrm{~nm}, 544 \mathrm{~nm}$ and $775 \mathrm{~nm}$ with oscillator strengths of $0.14,0.19$ and 0.13 , respectively. As a results, the new absorption of IM-PFB in ACN and THF at around $570 \mathrm{~nm}$ induced by $\mathrm{F}^{-}$or $\mathrm{CN}^{-}$should be ascribed to deprotonation of one $\mathrm{H}$ atom, not two $\mathrm{H}$ atoms.

Solvent polarity affects greatly the sensing ways of IM-PFB with anions. The possible interaction mechanism of IM-PFB with anions is outlined in Scheme 2. IM-PFB can form two adducts III and IV through hydrogen bond with one anion and two anions, respectively. III and IV would undergo a deprotonation to produce I and II in polar solvents. The binding stoichiometry of IM-PFB with $\mathrm{F}^{-}, \mathrm{H}_{2} \mathrm{PO}_{4}{ }^{-}$and $\mathrm{CN}^{-}$in various solvents was determined by Job's plots (Fig. S16†). Job's plot of IM-PFB shows the $1: 2$ binding stoichiometry in ACN and DCM, and the $1: 1$ binding stoichiometry in THF with anions. 


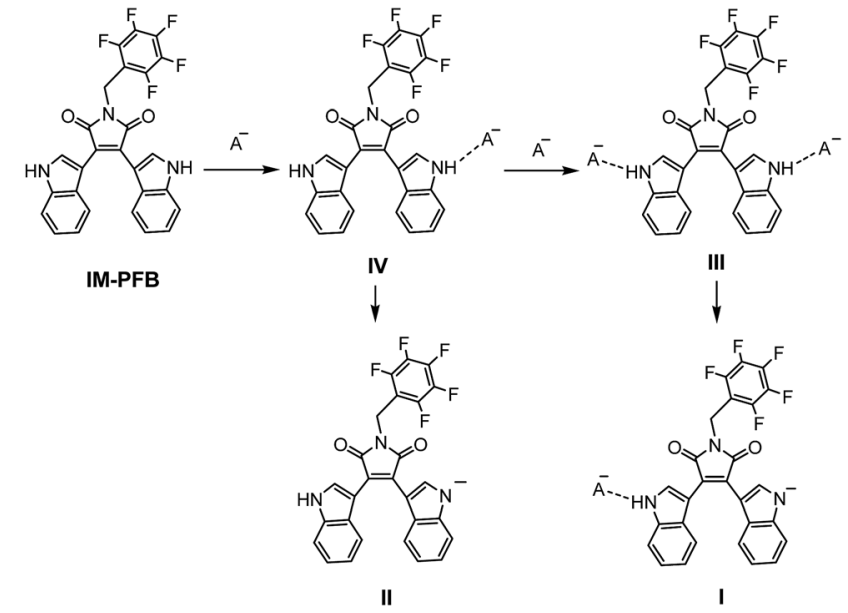

Scheme 2 Possible interaction mechanism of IM-PFB with anions $\left(A^{-}\right)$ in different solvents.

Based on the results, three interaction forms of IM-PFB with anions in different solvents are expected: (I in ACN; II in THF; III in DCM).

\section{Molecular logic operations}

The different interaction ways of IM-PFB with anions in different solvents arouse our interests in its applications in molecular logic system. Based on the distinct response to anions, IM-PFB molecules can be employed to construct various logic devices. First, the changes of peak intensity at $607 \mathrm{~nm}$ in emission $\left(I_{\mathrm{E}, 607}\right)$ and $570 \mathrm{~nm}$ in absorption $\left(I_{\mathrm{A}, 570}\right)$ in ACN (Fig. S17, ESI $\dagger$ ) are utilized as two outputs (O1 and O2) to design a 1-to-2 decoder and a 1:2 demultiplexer (Fig. S18 $\dagger$ ). If fluoride (50 eq.) is taken as only one input (In), the absence and presence of $\mathrm{F}^{-}$would cause the opposite results in $I_{\mathrm{E}, 607}(\mathrm{O} 2=1$ and 0$)$ and $I_{\mathrm{A}, 570}(\mathrm{O} 1=0$ and 1$)$ of IMPFB. As a result, a 1-to-2 decoder was obtained (Fig. S18a †). If IM-PFB and $\mathrm{F}^{-}$are defined as two inputs (In1 and In2), the function of $1: 2$ demultiplexer can be realized (Fig. S18b $\dagger$ ). When In2 (or address input) is set off, O1 $\left(I_{\mathrm{E}, 607}\right)$ reports the state of In1 (IM-PFB) and $\mathrm{O} 2\left(I_{\mathrm{A}, 570}\right)$ remains off, whereas when In2 is switched on, $\mathrm{O} 2$ replaces $\mathrm{O} 1$ to report the state of In1 and $\mathrm{O} 1$ turns off.

Secondly, as shown in Fig. S19 and S20 (ESI $\dagger$ ), IM-PFB in DCM can function as a 2-to-3 decoder by employing the stimuli of $\mathrm{F}^{-}$and $\mathrm{H}_{2} \mathrm{PO}_{4}{ }^{-}$as two inputs (In1 and In2), and the response of the emission intensity at $604 \mathrm{~nm}\left(I_{\mathrm{E}, 604}\right)$, the absorption intensity at $560 \mathrm{~nm}\left(I_{\mathrm{A}, 560}\right)$, and the ratio of absorption intensity at $467 \mathrm{~nm}$ and $350 \mathrm{~nm}\left(I_{\mathrm{A}, 467 / 350}\right)$ as three outputs (O1, O2 and O3). When no input was applied ( $\operatorname{In} 1=\operatorname{In} 2=0)$, IM-PFB exhibits strong orange emission ( $\mathrm{O} 1=1)$, no obvious absorption band at $560 \mathrm{~nm}(\mathrm{O} 2=0)$ and comparable absorption at $467 \mathrm{~nm}$ and $350 \mathrm{~nm}(\mathrm{O} 3=0)$. After In1 or In2 was applied, O1 $\left(I_{\mathrm{E}, 604}\right)$ was turned off for the fluorescence of IM-PFB in DCM quenched by $\mathrm{F}^{-}$or $\mathrm{H}_{2} \mathrm{PO}_{4}{ }^{-}$, but $\mathrm{O} 2\left(I_{\mathrm{A}, 560}\right)$ or $\mathrm{O} 3\left(I_{\mathrm{A}, 467 / 350}\right)$ was turned on for the change of absorption spectra in $560 \mathrm{~nm}$ or $350 \mathrm{~nm}$. a)
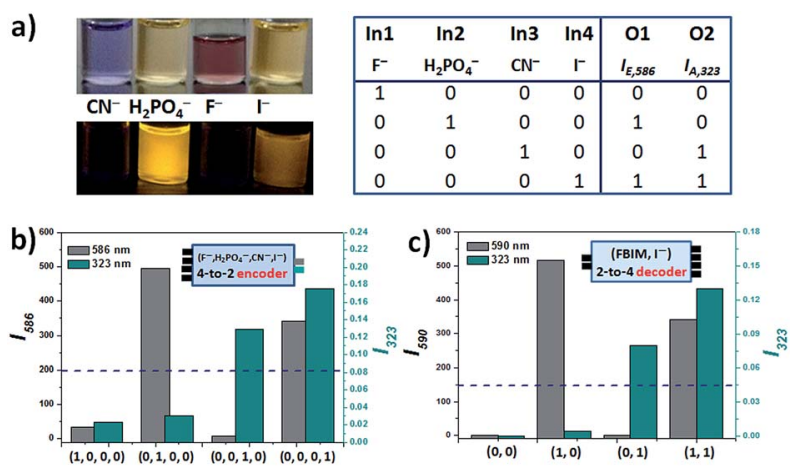

d)

\begin{tabular}{|cc|cccccc|}
\hline $\begin{array}{c}\text { In1 } \\
\text { FBIM }\end{array}$ & $\begin{array}{c}\text { In2 } \\
\mathrm{I}^{-}\end{array}$ & $I_{E, 586}$ & $\mathbf{I}_{\mathbf{A}, \mathbf{3 2 3}}$ & $\begin{array}{c}\mathbf{0 1} \\
\mathbf{( 0 , 0 )}\end{array}$ & $\begin{array}{c}\mathbf{O 2} \\
(\mathbf{0}, \mathbf{1})\end{array}$ & $\begin{array}{c}\mathbf{0 3} \\
(\mathbf{1}, \mathbf{0})\end{array}$ & $\begin{array}{c}\mathbf{0 4} \\
(\mathbf{1}, \mathbf{1})\end{array}$ \\
\hline 0 & 0 & 0 & 0 & 1 & 0 & 0 & 0 \\
1 & 0 & 1 & 0 & 0 & 1 & 0 & 0 \\
0 & 1 & 0 & 1 & 0 & 0 & 1 & 0 \\
1 & 1 & 1 & 1 & 0 & 0 & 0 & 1 \\
\hline
\end{tabular}

Fig. 7 4-to-2 encoder ( $a$ and b) and 2-to-4 decoder ( $c$ and d) demonstrated by the response of the emission intensity at $586 \mathrm{~nm}$ $\left(I_{E, 586}\right)$ and the absorption intensity at $323 \mathrm{~nm}\left(I_{\mathrm{A}, 323}\right)$ to $\mathrm{F}^{-}, \mathrm{H}_{2} \mathrm{PO}_{4}{ }^{-}$, $\mathrm{CN}^{-}$and $\mathrm{I}^{-}$in THF.

Last, IM-PFB in THF can mimic the logic operation of two reversible data-processing devices: 4-to-2 encoder and 2-to-4 decoder. When utilizing IM-PFB as a 4-to-2 encoder, the inputs are defined as 50 eq. of $\mathrm{F}^{-}$(In1), $\mathrm{H}_{2} \mathrm{PO}_{4}{ }^{-}$(In2), $\mathrm{CN}^{-}$(In3) and $\mathrm{I}^{-}(\mathrm{In} 4)$. The intensity of the fluorescence at $586 \mathrm{~nm}\left(I_{\mathrm{E}, 586}\right)$ and the absorptions at $323 \mathrm{~nm}\left(I_{\mathrm{A}, 323}\right)$ are considered as $\mathrm{O} 1$ and O2, respectively. As shown in Fig. S21 (ESI $\dagger$ ), the emission at $586 \mathrm{~nm}$ of IM-PFB can be significantly quenched by $\mathrm{F}^{-}$and $\mathrm{CN}^{-}$, not by $\mathrm{I}^{-}$and $\mathrm{H}_{2} \mathrm{PO}_{4}{ }^{-}$. This means that both In 1 and In 3 can turn $\mathrm{O} 1$ off, while In 2 and In 4 make $\mathrm{O} 1$ on. On the other hand, it is $\mathrm{CN}^{-}$and $\mathrm{I}^{-}$, not $\mathrm{F}^{-}$and $\mathrm{H}_{2} \mathrm{PO}_{4}{ }^{-}$that can induce a strong absorbance at $323 \mathrm{~nm}$. It means that $\mathrm{O} 2$ can be switched on by In 3 and In4. Thus, the truth table of the 4-to-2 encoder is represented as shown in Fig. 7. In addition, IM-PFB can be also easily reconfigured to behave as a molecular 2-to-4 encoder, when IM-PFB and $\mathrm{I}^{-}$act as two inputs (Fig. 7d). If $I_{\mathrm{E}, 586}$ and $I_{\mathrm{A}, 323}$ are also selected as two outputs, it was found that there are four different output results: $(0,0),(0,1),(1,0)$ and $(1,1)$. If taking the four output results as four kinds of new outputs (O1, O2, O3 and O4), the decoder function of IM-PFB can be demonstrated.

\section{Conclusions}

Bisindolylmaleimide derivatives (IMs) based on amine group as receptor were synthesized and found sensitive to anions with obvious colorimetric effect and fluorescence quenching. IM-PFB, IM-TBA and IM-MB with two twisted indole rings exhibit better selectivity for anions than IMC-MB with planar aromatic rings, due to their flexible recognition sites of $\mathrm{NH}$ groups. Interestingly, anion sensing of IM-PFB, IM-TBA and IM-MB behaves strong dependence on solvent types. For example, in ACN, $\mathrm{F}^{-}$can make IM-PFB form a new absorption 
peak at $564 \mathrm{~nm}$ and quench its emission at $609 \mathrm{~nm}$. In DCM, $\mathrm{H}_{2} \mathrm{PO}_{4}{ }^{-}$causes significant fluorescence quench of IM-PFB and little impact on its absorption. In $\mathrm{THF}, \mathrm{CN}^{-}$can induce the large response to both the absorption and emission of IM-PFB like $\mathrm{F}^{-}$in $\mathrm{ACN}$. $\mathrm{OH}^{-}$experiment and theory calculation indicate that the new absorption peak at around $570 \mathrm{~nm}$ is the result of deprotonation of one $\mathrm{H}$ atom. Based on the Job plots analysis, three possible interaction forms of IM-PFB with anions in different solvents are presented. Based on the anionsensing behavior of IM-PFB, three decoders (1-to-2, 2-to-3 and 2-to-4), a 4-to-2 encoder and a $1: 2$ demultiplexer have been developed. These results indicate IMs are good candidates for mimicking molecular logic computing.

\section{Acknowledgements}

This work was supported by the National Natural Science Foundation of China (21374017, 21401023 and 21574021), Specialized Research Fund for the Doctoral Program of Higher Education (20123503120002), Program for Innovative Research Team in Science and Technology in Fujian Province University (IRTSTFJ), the Natural Science Foundation of Fujian Province (2013H0018), and Educational Commission of Fujian Province (JA12059 and JA14068).

\section{Notes and references}

1 J. E. Bailey, M. Bohner, C. J. Brinker, B. Cornils, T. Evans, H. Greim, L. L. Hegedus, J. Heitbaum, W. Keim, A. Kleemann, G. Kreysa, J. Loliger, J. L. McGuire, A. Mitsutani, L. Plass, G. Stephanopoulos, D. Werner, P. Woditsch and N. Yoda, Ullmann's Encyclopedia of Industrial Chemistry, Wiley VCH, New York, 6th edn, 1999.

2 A. Bianchi, K. Bowman-James and E. Garcia-Espana, Supramolecular Chemistry of Anions, Wiley-VCH, New York, 1997.

3 E. R. Riegel and J. A. Kent, Riegel's handbook of industrial chemistry, Springer, 10th edn, 2003.

$4 \mathrm{~K}$. W. Kulig and B. Ballantyne, Cyanide toxicity, US Department of Health \& Human Services, Atlanta, GA, 1991.

5 B. L. True and R. H. Dreisbach, Dreisbach's handbook of poisoning, Parthenon Pub. Group, 2002.

6 P. Singh, M. Barjatiya, S. Dhing, R. Bhatnagar, S. Kothari and V. Dhar, Urol. Res., 2001, 29, 238-244.

7 Y. Marcus, J. Chem. Soc., Faraday Trans., 1991, 87, 2995-2999.

8 M. H. Keefe, K. D. Benkstein and J. T. Hupp, Coord. Chem. Rev., 2000, 205, 201-228.

9 A. S. Singh and S.-S. Sun, Chem. Commun., 2011, 47, 85638565.

10 M. S. T. Gonçalves, Chem. Rev., 2009, 109, 190-212.

11 M. Sun, S. Wang, Q. Yang, X. Fei, Y. Li and Y. Li, RSC Adv., 2014, 4, 8295-8299.

12 C.-Y. Hung, A. S. Singh, C.-W. Chen, Y.-S. Wen and S.-S. Sun, Chem. Commun., 2009, 1511-1513.

13 J. Andreasson and U. Pischel, Chem. Soc. Rev., 2010, 39, 174188.
14 A. P. de Silva and S. Uchiyama, Nat. Nanotechnol., 2007, 2, 399-410.

15 F. Pu, J. Ren and X. Qu, Adv. Mater., 2014, 26, 5742-5757.

16 P. A. de Silva, N. H. Q. Gunaratne and C. P. McCoy, Nature, 1993, 364, 42-44.

17 D. Margulies, G. Melman and A. Shanzer, Nat. Mater., 2005, 4, 768-771.

18 D. Margulies, G. Melman and A. Shanzer, J. Am. Chem. Soc., 2006, 128, 4865-4871.

19 J. Andreasson, U. Pischel, S. D. Straight, T. A. Moore, A. L. Moore and D. Gust, J. Am. Chem. Soc., 2011, 133, 11641-11648.

20 M. S. T. Gonçalves, Chem. Rev., 2009, 109, 190-212.

21 X. Mei, G. Wen, J. Wang, H. Yao, Y. Zhao, Z. Lin and Q. Ling, J. Mater. Chem. C, 2015, 3, 7267-7271.

22 J. Ling, B. Daly, V. A. D. Silverson and A. P. de Silva, Chem. Commun., 2015, 51, 8403-8409.

23 W.-L. Gong, M. P. Aldred, G.-F. Zhang, C. Li and M.-Q. Zhu, J. Mater. Chem. C, 2013, 1, 7519.

24 X. He, Z. Li, M. Chen and N. Ma, Angew. Chem., Int. Ed., 2014, 126, 14675-14678.

25 X. J. Jiang and D. K. Ng, Angew. Chem., Int. Ed., 2014, 53, 10481-10484.

26 M. Kumar, N. Kumar and V. Bhalla, Chem. Commun., 2013, 49, 877-879.

27 Y. Zhai, Z. Zhu, C. Zhu, J. Zhu, J. Ren, E. Wang and S. Dong, Nanoscale, 2013, 5, 4344-4350.

28 D. Kim and T. S. Lee, Chem. Commun., 2014, 50, 5833-5836.

29 R. Orbach, F. Remacle, R. D. Levine and I. Willner, Chem. Sci., 2014, 5, 1074-1081.

30 D. Tong, H. Duan, J. Wang, Z. Yang and Y. Lin, Sens. Actuators, B, 2014, 195, 80-84.

31 P. Remón, R. Ferreira, J.-M. Montenegro, R. Suau, E. PérezInestrosa and U. Pischel, ChemPhysChem, 2009, 10, 20042007.

32 A. K. Singh, P. K. Yadav, N. Kumari, R. Nagarajan and L. Mishra, J. Mater. Chem. C, 2015, 3, 12123-12129.

33 M. Li, Z. Guo, W. Zhu, F. Marken and T. D. James, Chem. Commun., 2015, 51, 1293-1296.

34 P. Singh, H. Singh, G. Bhargava and S. Kumar, J. Mater. Chem. C, 2015, 3, 5524-5532.

35 Z. Lin, Y.-D. Lin, C.-Y. Wu, P.-T. Chow, C.-H. g. Sun and T. Chow, Macromolecules, 2010, 43, 5925-5931.

36 Y.-S. Lee, Z. Lin, Y.-Y. Chen, C.-Y. Liu and T. J. Chow, Org. Electron., 2010, 11, 604-612.

37 Z. Lin, Y.-S. Wen and T. Chow, J. Mater. Chem., 2009, 19, 5141-5148.

38 T.-S. Yeh, T. J. Chow, S.-H. Tsai, C.-W. Chiu and C.-X. Zhao, Chem. Mater., 2006, 18, 832-839.

39 Z. Lin, Y. Ma, X. Zheng, L. Huang, E. Yang, C.-Y. Wu, T. J. Chow and Q. Ling, Dyes Pigm., 2015, 113, 129-137.

40 Z. Lin, H. C. Chen, S.-S. Sun, C.-P. Hsu and T. J. Chow, Tetrahedron, 2009, 65, 5216-5221.

41 N. Sarkar, K. Das, D. N. Nath and K. Bhattacharyya, Langmuir, 1994, 10, 326-329.

42 M. M. Faul, K. A. Sullivan and L. L. Winneroski, Synthesis, 1995, 12, 1511-1516. 
43 B. K. Kaletaş, C. Mandl, G. van der Zwan, M. Fanti, F. Zerbetto, L. De Cola, B. König and R. M. Williams, J. Phys. Chem. A, 2005, 109, 6440-6449.

44 K. Saita, M. Nakazono, K. Zaitsu, S. Nanbu and H. Sekiya, J. Phys. Chem. A, 2009, 113, 8213-8220.
45 D. Aldakov and J. P. Anzenbacher, Chem. Commun., 2003, 1394-1395.

46 P. Anzenbacher Jr, K. Jursikova, D. Aldakov, M. Marquez and R. Pohl, Tetrahedron, 2004, 60, 11163-11168. 